\title{
Origins of the deficiency of Amazonian soils - a new approach
}

\author{
Rolf Singer \\ Instituto Nacional de Pesquisas \\ da Amazônia, Manaus
}

When the primary terra firme forest which covers most of the Amazonian area is destroyed to permit agricultural activity, the soil deteriorates rapidly under the influence of physical conditions of the environment with concurrent changes in its microbiology (Batista, 1966). It is well known (Olah et al. 1978) that aside from the environmental influences after deforestation, the characteristics of the biodegradation and humification previous to deforestation have a profound effect upon eventual soil degradation. The primary terra firme forest, in spite of an ample supply of fallen leaves and dead wood has underneath this freshly fallen litter only an extremely thin or non-existent humus layer, supported by clay which is much poorer in organic matter.

Year-round quantitative studies - now completed - on litter decomposing fungi and ectotroph (') formation in the Amazonian forests at INPA, Manaus, show that:

1. the litter decomposing non-mycorrhizal saprophytic fungi are in overall quantity, diversity (and thus adaptation to different substrata), completeness of coverage of the forest ground, and continuity of activity during the entire year (including the "dry" season), greatly favored in the terra firme when compared to other forest communities, particularly the ectotroph-dominated forests;

2. the undisturbed primary terra firme forest is almost to entirely devoid of ectotrophs whereas the shaded campina and campinarana vegetation (see Anderson et al., 1975) under equal climatic conditions is ectotroph-dominated (as are tropical-montane Alneta and Querceta and most temperate forest (cf. Singer \& Morello, 1960; Singer, 1971):
3. the ectotroph forests, including the campinarana, having only one forth to one fifth of the litter-decomposing fungi per $\mathrm{m}^{2}$, are characterized by a high degree of raw humus accumulation so that this layer reaches considerable depth whereas in the terra firme forest it is extremely thin to almost nonexistent.

These data confirm results of experimental work by Gadgil \& Gadgil (1971) showing increased litter decomposition in the absence of mycorrhizal (apparently ectomycorrhizal) fungi; they are also in agreement with Harley's concept of shortcycling of mineral nutrients. especially the critical $\mathrm{P}$ and $\mathrm{N}$ compounds, by mycorrhizal fungi.

It cannot be denied that other factors (invertebrates, endomycorrhizae) in the extremely complex ecosystems of the wet tropics might contribute to or accentuate the differences observed in the humus formation in Amazonian forests. It is nevertheless reasonable to conclude that the absence or scarcity of ectotrophs in the terra firme forest increase the rate of litter decomposition per unit time to such a degree that no significant humus cover can be accumulated.

This explanation opens up perspectives for more rational planning of agricultural development in Amazonia (and other regions with anectotrophic tropical lowland forests) by introducing ectotrophically mycorrhizal trees before complete deforestation - a procedure technically as well as economically feasible. It is this perspective that justifies the present preliminary note.

\section{Resumo}

Considera-se que a deficiência dos solos amazônicos, após o corte da floresta de terra firme, seja cau-

(1) - Ectotroph is the complex organism of ectotrophically mycorrhizal tree and ectotrophically mycorrhizal fungi seen as an element of a forest community (Singer \& Morello, 1960). 
sada, principalmente, pela ausência de-vegetação dominante ectotrófica, que favorece a atividade de fungos que decompõem a liteira a um tal grau que não há acumulação de uma camada significante de húmus fresco,

\section{BIBLIOGRAFIA CITADA}

Anderson, A. B.; Prance, G. T. \&

ALBUQUeRQue, B. W. P.

1975 - Estudos sobre a vegetaçăo das campinas amazônicas. II. Acta Amazonica, 5(3) : 225-242.

Batista, A. Chaves

1960 - Exposição sobre solos amazônicos. In : Simpósio sobre a Biota Amazônica, Belém. Exposição do IMUFPe (unpublished) cf Sin- ger, R. 1969, A. Chaves Batista. Sydowia, $22: 344$.

GADGIL, R. L. \& Gadgil, G. A.

1971 - Mycorrhiza and litter decomposition. Nature, $233: 113$.

Oláh, G. M.; Reisinger, O. \& Kilbertus, G. 1978 - Biodégradation et humification. Montreal.

SINGER, R.

1971 - Forest mycology and forest communities in South America. II. In: Hacskaylo, E. Mycorrhizae. Washington, U, S. Gov. Printing Office. p. 204-215.

Singer, R. \& Morello, J, H.

1960 - Ectotrophic tree mycorrhiza and forest communities. Ecology, 41: 549-551.

(Aceito para publicação em $08 / 06 / 78$ )

\title{
Nota prévia sobre o "pão do índio" da Amazônia Brasileira
}

\author{
Izonete de Jesus da Silva Araujo \\ Instituto Nacional de Pesquisos \\ da Amazônia, Manaus \\ Maria Alves de Sousa \\ Universidade Federal da Paraiba
}

O "pão do índio" é amplamente distribuído na Amazônia, onde encontra condições favoráveis ao seu desenvolvimento, chegando a atingir mais de $60 \mathrm{~kg}$ (Maravalhas, 1965). É subterrâneo, o que torna difícil sua coleta, sendo encontrado principalmente por leigos nas escavações para construções de estradas e arações para lavouras. Até o momento, temos registros de sua ocorrência nos Estados do Amazonas, Mato Grosso, Pará e Territórios de Rondônia e Roraima.

O organismo em estudo é um "milita" (grande esclerócio) que pertence ao gênero imperfeito, Pachyma Fries. O esclerócio é muito pesado, tuberoso, comestível. Segundo Maravalhas (1965), contém muito material de reserva, predominando polissacarídeos. É constituído por hifas que formam uma massa compacta, vítrea, opaca, muito dura quando seca, mucilaginosa em $\mathrm{KOH}$, e variegada de cores, correspondendo a Locquin (1975), mais ou menos "albus" (H8h) a "russus" (A2C) muito claro ou escuro. A região periférica é constituída por uma camada de hifas laxas de cor ho- mogênea, "pudorinus" (Q5h), sobre a qual forma-se uma crusta delgada, "venetus" (Z1b) escuro e quebradiça (Fig. 1). O sistema hifal é dimítico com hifas geradoras e esqueléticas. Hifas geradoras com $3-7 \mu \mathrm{m}$ de diâmetro, hialinas, de paredes delgadas, às vezes levemente espessadas, com fíbulas, septos não muito freqüentes, não ramificadas ou ramificadas. Quando ramificadas apresentam uma ramificação longa ou várias ramificações curtas. Algumas hifas, apresentam pequenas $\mathrm{e}$ abundantes granulações, que coram intensamente em azul de algodão. Hifas esqueléticas medindo $4,2-15 \mu \mathrm{m}$ de diâmetro, hialinas, de paredes espessadas, ápices obtusos, septos e ramificações não observados. Estas hifas quando observadas em $\mathrm{KOH}$ apresentam as paredes mais nitidamente visíveis e as extremidades agudas e freqüentemente curvas.

Os basidiocarpos analisados foram obtidos em laboratório, sob a orientação do Dr. Rolf Singer, o qual colocou um esclerócio num balde galvanizado com solo humoso conservado umedecido. Após 2 meses observamos, 